\title{
Downregulation of MDC1 and 53BP1 by short hairpin RNA enhances radiosensitivity in laryngeal carcinoma cells
}

\author{
QIHENG GOU $^{1 *}$, YUXIN XIE ${ }^{2 *}$, LEI LIU $^{2 *}, \mathrm{KEQI} \mathrm{XIE}^{3}$, YANXIA WU $^{4}$, \\ QIANQIAN WANG ${ }^{2}, \mathrm{ZHU} \mathrm{WANG}^{4}$ and PING $\mathrm{LI}^{2}$
}

\begin{abstract}
${ }^{1}$ State Key Laboratory of Biotherapy/Collaborative Innovation Center of Biotherapy, West China Hospital, Sichuan University, Chengdu, Sichuan 610041; ${ }^{2}$ Cancer Center, West China Hospital, West China Medical School, Sichuan University, Chengdu,

Sichuan 610041; ${ }^{3}$ Department of Anesthesiology, Mianyang Central Hospital, Mianyang, Sichuan 621000;

${ }^{4}$ Laboratory of Molecular Diagnosis of Cancer, West China Hospital,

Sichuan University, Chengdu, Sichuan 610041, P.R. China
\end{abstract}

Received October 26, 2014; Accepted February 10, 2015

DOI: 10.3892/or.2015.3980

\begin{abstract}
DNA double-strand breaks (DSBs) induced by ionizing radiation (IR) are among the most cytotoxic types of DNA damage. The DNA damage response (DDR) may be a reason for the cancer cell resistance to radiotherapy using IR. Identified as critical upstream mediators of the phosphorylation of ataxia telangiectasia-mutated (ATM) pathway, mediator of DNA damage checkpoint 1 (MDC1) and p53-binding proteins 1 (53BP1) may affect the radiosensitivity of tumor cells. In the present study, we generated two HEP-2 cell lines with a stable knockdown of $M D C 1$ or $53 B P 1$ with short hairpin RNA (shRNA), respectively, and investigated the effect of MDC1 and 53BP1 on cell radiosensitivity, cell cycle distribution and the formation of cell foci. Downregulation of the two proteins reduced the number of clonogenic cells that treated with IR. Accumulation of G2/M phase cells was detected after the MDC1 and 53BP1 downregulation. These results indicated that the expression of MDC1 or 53BP1 limited tumor cell sensitivity to radiotherapy and may play an important role in the DNA repair progression. Furthermore, the MDC1 foci was identified and presented in the 53BP1-inhibited cells. By contrast, the 53BP1 foci was absent from the MDC1-inhibited cells. The results confirmed that the recruitment of 53BP1 into the foci occurred in an MDC1-dependent manner.
\end{abstract}

\section{Introduction}

DNA double-strand breaks (DSBs) arising from a number of endogenous and exogenous sources, such as oxidative stress

Correspondence to: Professor Ping Li, Cancer Center, West China Hospital, West China Medical School, Sichuan University, Gaopeng Street, Keyuan Road 4, Chengdu, Sichuan 610041, P.R. China E-mail: leepingmd68@163.com

*Contributed equally

Key words: DNA double-strand breaks, DNA damage checkpoint 1, p53-binding proteins 1 , radiosensitivity, arrest of cell cycle and ionizing radiation (IR), can induce the DNA damage response (DDR) (1). As an important mechanism of maintenance of genomic integrity, the DDR cascade senses genome damage and activates several downstream pathways including cell cycle checkpoints and DNA repair, to protect cancer cells from DNA damage. Ataxia telangiectasia-mutated (ATM) and ATM-related (ATR) kinases are phosphoinositol 3-kinase-like kinases (PIKKs) that regulate the DNA damage signaling response $(2,3)$. Once activated, ATM and ATR in turn phosphorylate the histone variant $\mathrm{H} 2 \mathrm{AX}$ and downstream effectors, including the Chk1 and Chk 2 cell cycle kinases, resulting in cell cycle checkpoint activation and cell cycle arrest. The checkpoint activation frequently leads to modifications of DNA repair factors and results in a more efficient removal of the lesions and increased resistance to further damage. The cell cycle arrest of cancer cells induced by IR damage is the main reason of radioresistance (4).

An emerging classification of proteins termed mediators participates in the transduction of the checkpoint response (5). These proteins are thought to be involved in the recognition of DNA damage and the recruitment of additional proteins that facilitate downstream signaling and repair. Important mediators are mediators of DNA damage checkpoint 1 (MDC1) and p53 binding protein 1 (53BP1), which participated in the signaling pathway initiated by ATM $(6,7)$. MDC1, containing functional domains such as forkhead-associated domain (FHA) and BRCA1 carboxy-terminal (BRCT) domains, can recognize the phosphorylated $\mathrm{H} 2 \mathrm{AX}$ proximal to the lesion via its tandem BRCT domain and help to counter $\mathrm{H} 2 \mathrm{AX}$ dephosphorylation. As a mediator protein, MDC1 is localized to the damage site independently of ATM but is recruited to the damage site through interaction with ATM-phosphorylated histone $\mathrm{H} 2 \mathrm{AX}$ and distributed along the damaged region $(8,9)$. MDC1-H2AX interaction is important for the accumulation of other DDR proteins such as Nijmegen breakage syndrome 1 (NBS1), 53BP1 and the activated ATM to the DSBs site, thus amplifying DNA damage signals of G2/M phase checkpoint activation. 53BP1, whose efficient accumulation at sites of DSBs requires $\mathrm{H} 2 \mathrm{AX}$ and a functional MDC1-H2AX, subsequently undergoes a notable relocalization to the damage 
regions following exposure to DSB-inducing IR $(10,11)$. The cell response to DSBs can be characterized by the formation of foci by fluorescence microscopy. The foci represent the DSBs sites and contain certain DDR proteins such as BRCA1, 53BP1, nuclear factor with BRCT domain 1 (MDC1/NFBD1), NBS1, microcephalin (MCPH1), pax transactivation domain-interacting protein (PTIP) and topoisomerase II $\beta$ binding protein (TopBP1) (12-15). The foci formation has been functionally involved in the processes of DNA repair and checkpoints activation. As key constituents of IR-inducing foci, MDC1 and 53BP1 were shown to maintain cell cycle arrest and promote to repair pathways (11).

IR and many chemotherapies cause DNA damage that has the potential to kill cells (16). The responses of tumor cells to such damage, including activation of the cell cycle checkpoint and DNA repair, is a determinant of cancer cell sensitivity to radiotherapy. Previously, it was found that deficiency or abnormality of the DDR-related proteins affected the DNA repair progress, activation of the cell cycle checkpoint and cell death pathways, thereby influencing the radiosensitivity of tumor cells (17). As an indispensable component of DDR, MDC1 and 53BP1 have been reported to be abnormally expressed in head and neck tumor cell line Hep-2 (18). However, whether they have an impact on the cell cycle checkpoint of Hep-2 treated with IR remains to be determined. In the present study, we downregulated MDC1 and 53BP1 using short hairpin RNA (shRNA) to determine the relationship between MDC1 and 53BP1 expression and Hep-2 sensitivity to IR. We also discussed the roles of MDC1 and 53BP1 in the activation of each checkpoint.

\section{Materials and methods}

Cell line and culture. Human Hep-2 laryngeal carcinoma cells were kindly provided by the State Key Laboratory of Biotherapy, Sichuan University (Sichuan, China). The cells were cultured in DMEM or RPMI-1640 (Gibco-BRL, Gaithersburg, MD, USA) supplemented with $10 \%$ fetal bovine serum (FBS) and penicillin/streptomycin in a humidified incubator with $5 \% \mathrm{CO}_{2}$ and $95 \%$ air at $37^{\circ} \mathrm{C}$.

shRNA design and plasmid construction. The targeting sequences for shRNA plasmids were as follows: hMDC1 sh1, 5'-GCAGAAGCCAATCAGCAAA-3'; sh2, 5'-AGAGGGAC AATGATACAAA-3'; h53BP1 and sh1, 5'-GATACTGCCTCA TCACAGT-3'; h53BP1 and sh2, 5'-GAACGAGGAGACGGT AATA-3'. The negative control sequence (NC) used was 5'-TTCTCCGAACGTGTCACGTT-3'. The targeting fragments were introduced into GV102 plasmid (GeneChem Inc., Shanghai, China).

Establishment of stable cell lines. The shRNA plasmids were transfected into the Hep-2 cell line with Lipofectamine ${ }^{\mathrm{TM}} 2000$ (Invitrogen Life Technologies, Carlsbad, CA, USA) according to the manufacturer's instructions. Briefly, $1.5 \times 10^{5}$ cells were seeded into a 24 -well plate for $24 \mathrm{~h}$ before transfection in order to achieve $90-95 \%$ confluency at the time of transfection. For each transfection sample, $0.8 \mu \mathrm{g}$ plasmid DNA and $2 \mu \mathrm{l}$ lipofectin were diluted in $50 \mu \mathrm{l}$ Opti-MEM and incubated for 5 min. Diluted Lipofectamine ${ }^{\mathrm{TM}} 2000$ was then combined with this diluted DNA and incubated for $20 \mathrm{~min}$ at room tempera- ture, and this mixture was subsequently overlaid onto the cells and incubated for $6 \mathrm{~h}$. Then, $2 \mathrm{ml} /$ well growth medium (10\% FBS) was added to further cultivate the cancer cells for an additional $24 \mathrm{~h}$. The transfected cells were then transferred at a 1:10 dilution into a 6-well plate where stably transfected Hep-2 cells were selected after being cultivated in the presence of G418. Following selection, stable cell populations were identified by direct immunofluorescence and designated as NC (transfected by sh-Negative control), C6 (transfected by sh-MDC1) and P6 (transfected by sh-53BP1) The interference efficiency of shRNAs was determined through green fluorescent protein (GFP) and western blot analysis. The GFP was used as a marker of the plasmid expression.

Assessment of the cell cycle phase using flow cytometry. A cell cycle assay was performed using flow cytometry (FCM). Briefly, the cells in the logarithmic growth phase were released from the culture flask using $0.25 \%$ trypsin and dispersed in RPMI-1640 medium with 10\% FBS. The cell suspension was then centrifuged for $10 \mathrm{~min}$ at $1000 \mathrm{rpm}$, and the supernatant was discarded. The cells were washed and resuspended twice at $1 \times 10^{6}$ cells $/ \mathrm{ml}$ in PBS (pH 7.4) followed by fixation with $70 \%$ ethanol at $4^{\circ} \mathrm{C}$ for $1 \mathrm{~h}$. After washing, the cells were incubated with 50 and $250 \mu \mathrm{g} / \mathrm{ml} \mathrm{RNase}$ at $4^{\circ} \mathrm{C}$ for $30 \mathrm{~min}$ and analyzed by FCM. Samples were used in triplicate for each group.

Western blotting. Cells were collected with PBS and lysed at $4^{\circ} \mathrm{C}$ in non-reducing cell lysis buffer. Each sample containing an equal amount of whole cell lysates was subjected to electrophoresis in 6\% SDS-polyacrylamide gels. After electrophoresis at $170 \mathrm{~V}$ for $2 \mathrm{~h}$, the protein was blotted onto a PVDF membrane at $100 \mathrm{~V}$ for $2 \mathrm{~h}$. Primary antibodies against MDC1 (1:1,000; Abcam Inc., Cambridge, MA, USA), 53BP1 (1:1,000; Cell Signaling Technology, Danvers, MA, USA) and $\beta$-actin (1:1,000; Sigma-Aldrich, St. Louis, MO, USA) were used, according to the manufacturer's instructions. After washing the membrane, the secondary antibody (HRP-conjugated anti-mouse $\mathrm{IgG}$ ) was used for the detection of MDC1, 53BP1 and $\beta$-actin. The bands were visualized by the ECL detection system after washing the membrane.

Immunofluorescence. Cells grown on coverslips were washed with PBS, fixed and permeabilized with $4 \%$ paraformaldehyde and $0.1 \%$ Triton X-100 and washed three times with PBS. The cells were incubated with $5 \%$ normal goat serum at $4^{\circ} \mathrm{C}$ for $1 \mathrm{~h}$ followed by incubation with anti-53BP1 (1:200; Cell Signaling Technology) and anti- MDC1 (1:400; Abcam Inc.) antibodies which was diluted with PBS containing 5\% normal goat serum. After washing with PBS, the cells were incubated with FITC-conjugated goat anti-rabbit antibodies and then incubated with $0.5 \mu \mathrm{g} / \mathrm{ml}$ Destination Access Point Identifier (DAPI) (1:200; Sigma-Aldrich). The cells were washed to remove unbound antibodies and DAPI and observed using a confocal microscope.

Proliferation curve assay. The cells from each experimental group were plated at $1 \times 10^{3}$ cells/well on 96-well plates. Cell proliferation was assessed by the MTT (moto-nuclear cell direc cytotoxicity assay) [3-(4,5-dimethylthiazol-2-yl)-2,5-diphenyltetrazoliumbromide] assay at the indicated time-points 
A

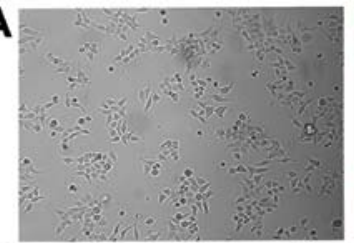

B

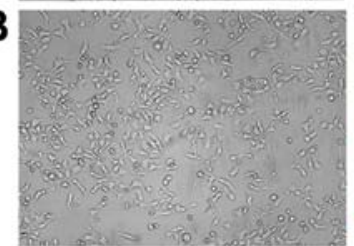

C

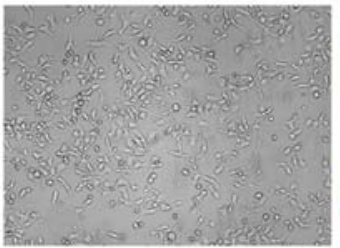

D

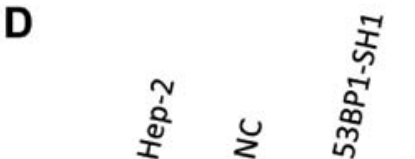

53BP1

$\beta$-actin

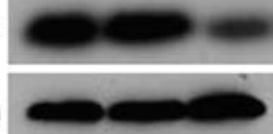

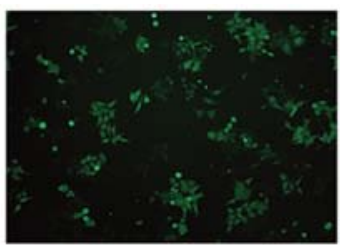
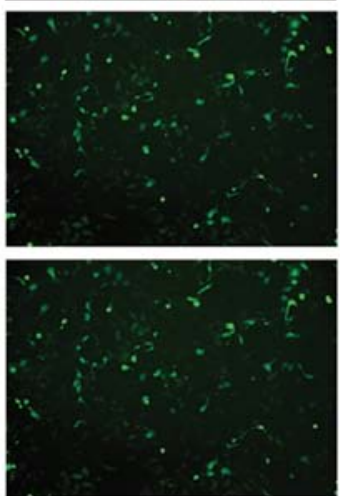

E

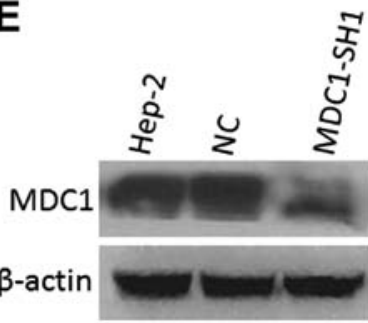

Figure 1. Light microphoto and fluorescent expression in Hep-2-transfected stable cell lines. (A) C6 (transfection of pMDC1), (B) P6 (transfection of p53BP1), (C) NC (transfection of pNegative-control). Level of protein of (D) P6 and (E) C6 detected by western blotting. MDC1, mediator of DNA damage checkpoint $1 ; \mathrm{NC}$, negative control.

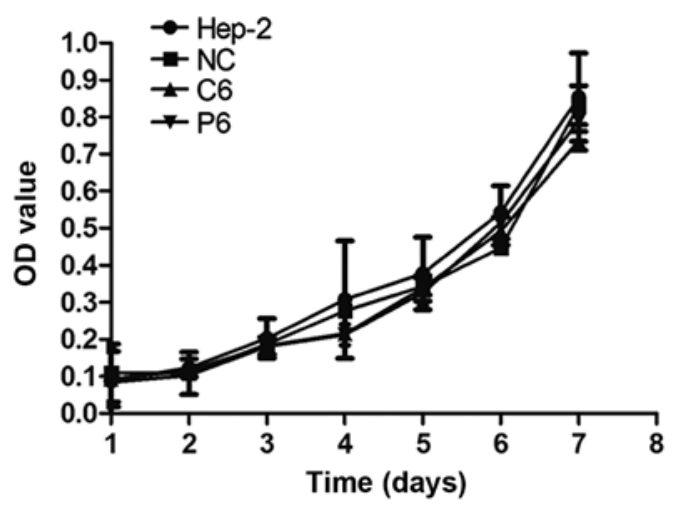

Figure 2. The cell viability in each group. Cell proliferation was rarely suppressed in each group, while transfection with plasmid and downregulation of MDC1 and 53BP1 had no inhibitory effect on Hep-2 cells. MDC1, mediator of DNA damage checkpoint 1; 53BP1, p53-binding proteins 1 .

$(1,2,3,4,5,6$, and 7 day). Briefly, $20 \mu 1$ of MTT $(5 \mu \mathrm{g} / \mathrm{ml})$ was added to each well and incubated for $2 \mathrm{~h}$. Subsequently, the MTT solution was removed, and $150 \mu \mathrm{l}$ of dimethyl sulfoxide (DMSO) was added to each well and the plate was agitated for $10 \mathrm{~min}$ to dissolve the formazan crystals. The absorbance of each well was determined at $570 \mathrm{~nm}$ on a microtiter plate reader (BioTek, Winooski, VT, USA).

Detection of IR sensitivity by colony formation assays. Briefly, the cell lines were plated at an appropriate density $\left(10^{1}, 10^{2}, 10^{3}, 10^{4}\right.$ and $10^{5} /$ well $)$ on 6-well plates and incubated for $5 \mathrm{~h}$ at $37^{\circ} \mathrm{C}$. The cells were exposed to X-ray at a single dose of $0,2,4,6,10$ Gy followed by incubation for 14 days without disturbance. Formed colonies were fixed by methanol for $15 \mathrm{~min}$ and visualized by staining with Giemsa. The plating efficiency and surviving fraction were evaluated as:

Plating efficiency $(\mathrm{PE})=\frac{\text { no. of control group colonies formed }}{\text { no. of cells seeded }} \times 100 \%$

Surviving fraction $(\mathrm{SF})=\frac{\text { no. of experimental group colonies }}{\text { no. of cells seeded } \mathrm{x} \text { PE }}$

Statistical analysis. Statistical analysis was carried out using the SPSS 15.0 software (SPSS, Inc., Chicago, IL, USA). Survival curves were generated based on the Kaplan-Meier method and the statistical significance was determined by Mann-Whitney U-tests. A P-value $<0.05$ on a two-tailed test was considered statistically significant.

\section{Results}

Establishment of cell lines with a low expression of MDC1/ $53 B P 1$. To determine whether a decrease in $M D C 1 / 53 B P 1$ expression was affected by $M D C 1 / 53 B P 1$ gene silencing, we generated laryngeal carcinoma Hep-2 cells with a stable expression of shRNAs targeting $M D C 1 / 53 B P 1$. The stable cell lines were designated as C6 and P6, respectively, (Fig. 1A and B). The cell line with a stable expression of sh-Negative control was designated as NC (Fig. 1C). The downregulation of MDC1 and 53BP1 was assessed by western blotting. Data showed that the protein expression was decreased significantly in shRNA-transfected cells compared with shNC-transfected cells $(\mathrm{P}<0.05)$. However, no significant difference was detected between the $\mathrm{NC}$ and untransfected cells $(\mathrm{P}>0.05)$ (Fig. 1D and E).

Downregulation of MDC1/53BP1 is unrelated to cell proliferation. MTT assays were performed to assess the effect of $M D C 1 / 53 B P 1$ downregulation on cell proliferation. The cells transfected with sh-MDC1 or sh-53BP1 showed the same proliferation ability as the untransfected cells $(\mathrm{P}>0.05)$. The results suggested that the downregulation of 53BP1 or MDC1 and the expression of shRNAs had no effect on cell proliferation (Fig. 2).

Downregulation of MDC1/53BP1 increases laryngeal carcinoma cell radiosensitivity. To investigate whether the downregulation of MDC1 or 53BP1 was associated with an increase in radiosensitivity, cells with a stable expression of shMDC1 or sh53bp1 were subjected to the colony formation assay following exposure to different doses of X-rays.Compared to the parental Hep-2 cells, the MDC1/53BP1 downregulation of Hep-2 cells exhibited a significant increase in sensitivity to the $\mathrm{X}$-rays. The colony formation in the NC cells (transfected with control shRNA) was $56.7 \%$ for $2 \mathrm{~Gy}$. By contrast, the X-rays seemed to exert more effect on the C6 and P6 cells with the downregulation of $\mathrm{MDC} 1$ or 53BP1, with the efficacy of colony formation being reducing from 43.5 to $48.4 \%$. A statistically 


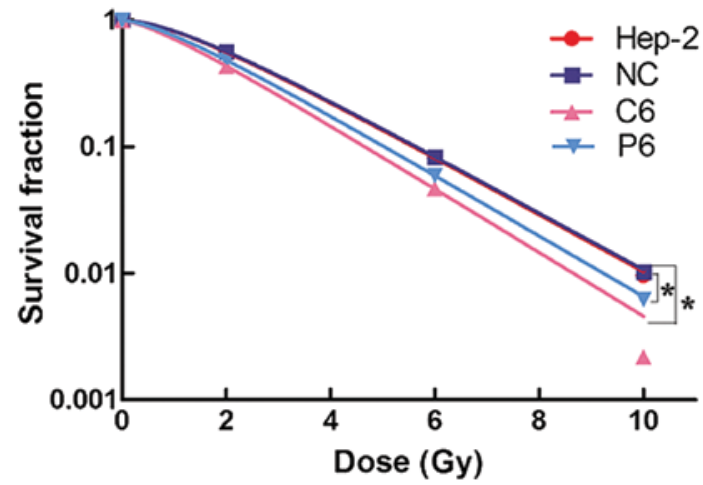

Figure 3. Clonogenic survival curves of different cell lines measured after 14-day with clone formal assay after IR. Table I shows the factor of radiosensitivity of the different cell lines. ${ }^{*} \mathrm{P}<0.05$ vs. Hep-2. IR, ionizing radiation.

significant difference $(\mathrm{P}=0.02<0.05)$ was found in the colony formation when C6 and P6 cells were compared with the NC or parental cells (Fig. 3). Furthermore, a significant decrease in Dq and D0, which are important indicators of cell radiosensitivity, was observed when the MDC1- and 5BP1-inhibited cells were compared with the parental cells $(0.721,0.9$ vs. 1.226 and $1.724,1.811$ vs. 1.92) $(\mathrm{P}<0.05)$ (Table I) (19). The effect of MDC1 and 53BP1 knockdown on the cell IR sensitivity showed an increase in a dose-dependent manner. These data indicated that the downregulation of MDC1 and 53BP1 levels in laryngeal carcinoma Hep-2 cells increased the sensitivity to IR.

Effects of $M D C 1$ and 53BP1 on cell cycle arrest. To determine whether $\mathrm{MDC} 1$ and 53BP1 play a role in the activation of DSB cell cycle checkpoints, we analyzed the effects of MDC1 and 53BP1 downregulation with irradiation, on the cell cycle distribution using FCM. Twelve hours after exposure to a low dose of radiation (2 Gy), the variations of intra-S-phase cells among the four groups showed no significant difference $(\mathrm{P}>0.05)$. However, C6 showed a significant increase in the proportion of $\mathrm{G} 2 / \mathrm{M}$ phase cells and a reduction in the proportion of $\mathrm{G} 0 /$ G1 phase cells compared with the NC cells. P6 showed a 5-fold increase in the percentages of cells in the $\mathrm{S}$ phase compared with the NC or parental cells. After treatment with $6 \mathrm{~Gy}$ X-rays, the percentage of $\mathrm{C} 6$ cells in $\mathrm{G} 2 / \mathrm{M}$ was double that of the control cells. P6 cells in the $\mathrm{S}$ phase were markedly reduced and significantly increased in $\mathrm{G} 2 / \mathrm{M}$ phase $\mathrm{P} 6$ cells were detected. The percentages of P6 cells in the G2/M were 3-fold higher than those in the control group. Under a high dose of IR (10 Gy), no changes in the number of $\mathrm{P} 6$ cells in the $\mathrm{S}$ and $\mathrm{G} 2 / \mathrm{M}$ phase were observed, however, notable changes were evident in the C6 cells. Hardly any C6 cells in intra-S phase were detected. By contrast, 5-fold more C6 cells compared with the control groups were identified in $\mathrm{G} 2 / \mathrm{M}$ phase. Twenty-four hours after the X-ray treatment, the $\mathrm{NC}$ and parental cells recovered the cell cycle distribution similar to that of their corresponding non-X-ray-treated cells. There was no difference in the cell cycle distribution of $\mathrm{C} 6$ and $\mathrm{P} 6$ between the cell populations analyzed 12 or $24 \mathrm{~h} \mathrm{X}$-rays were performed (Fig. $4 \mathrm{~B}$ and C).

To determine the role of MDC1 and 53BP1 to the cell response of IR, we examined the level of MDC1 and 53BP1 before and after IR treatment by western blotting (Fig. 4A). After $12 \mathrm{~h}$ ofexposure to IR, an increase in MDC1 and 53BP1
Table I. The radiosensitivity parameters $(\overline{\mathrm{x}})$ of Hep-2 and transfected cells.

\begin{tabular}{lcccc}
\hline Cell lines & \multicolumn{1}{c}{ D0 } & $\mathrm{N}$ & $\mathrm{Dq}$ & $\mathrm{SF} 2$ \\
\hline Hep-2 & 1.920 & 1.893 & 1.226 & 0.561 \\
NC & 1.928 & 1.911 & 1.249 & 0.567 \\
C6 & $1.724^{\mathrm{a}}$ & 1.519 & $0.721^{\mathrm{a}}$ & 0.435 \\
P6 & $1.811^{\mathrm{a}}$ & 1.644 & $0.900^{\mathrm{a}}$ & 0.484 \\
\hline
\end{tabular}

${ }^{\text {a }} \mathrm{P}<0.05$ vs. Hep-2 and NC groups; D0, mean lethal dose; N, extrapolation number; Dq, quasi-domain; SF2, survival fraction at 2 Gy.

expression was detected in the shNC- and shRNA-transfected cells. The expression of MDC1 and 53BP1 increased in an IR dose-dependent manner.

Formation of $\mathrm{MDCl}$ and 53BP1 foci in $\mathrm{MDCl}$ and 53BP1inhibited cells. As critical upstream mediators of DDR, MDC1 and 56BP1 could be recruited to the sites of DSBs induced by IR and thus resulting in the formation of foci (20). The foci were visualized indirectly through immunostaining with antibodies against the protein of interest (21). The antibodies were used directly against $\mathrm{MDC} 1$ and were used to detect the IR-induced foci formation of $\mathrm{MDC1}$ and 53BP1 (Fig. 5). After exposure to $\mathrm{X}$-rays, a large number of $\mathrm{MDC} 1$ and 53BP1 foci were detected in Hep-2 and NC cells, with only slight variation. The MDC1 foci were also detected in p53BP1-knockdown P6 cells and the number was influenced by the irradiation dose. However, the disappearance of 53BP1 foci was identified in IR-treated P6 cells. The MDC1 and 53BP1 foci were undetectable in C6 cells with a downregulation of MDC1 after X-ray treatment.

\section{Discussion}

The mechanisms of normal cells include repair system and kinase-dependent signaling pathways that delay and arrest cell cycle progression to maintain genomic integrity $(1,18)$. By contrast, essential functional molecules used to repair IR-induced damage and arrest the cell cycle, thereby providing the amount of time necessary for DNA repair are often deficient in tumor cells. Moreover, such tumor cells die of radiation-related DNA damage because this damage is difficult to repair $(8,13)$. As important mediators of the DDR signaling pathway, MDC1 and 53BP1 play a role in the activation of cell checkpoint, tumorigenesis and tumor invasion in many mammalian cells $(7,22,23)$. Early studies have reported that MDC1 and 53BP1 abnormal expression in human laryngeal carcinoma Hep-2 cells (18). To further confirm whether the expression of MDC1 and 53BP1 in Hep-2 has a causative function in the cell resistance to IR, we established stable Hep-2 cell lines with downregulation of MDC1 and 53BP1, respectively. MDC1 and 53BP1 were dispensable for proliferation and cell cycle in normal cell as well as in MDC1- or 53BP1-inhibited cells when no difference in cell proliferation or the cell cycle between the parental and stable-transfected cells was identified. This observation suggests that the two proteins may only be important when damage is caused by exogenous factors, such as radiation $(17,24,25)$. 
A

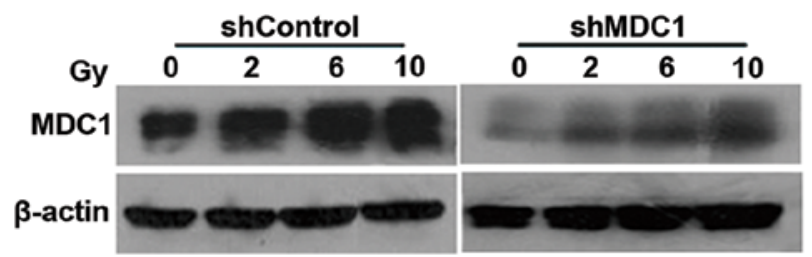

B

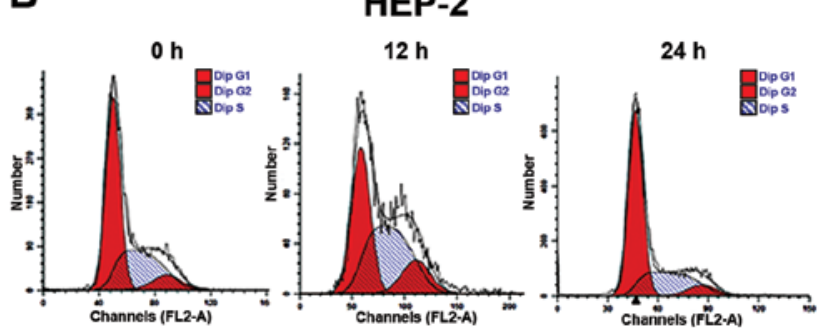

C6

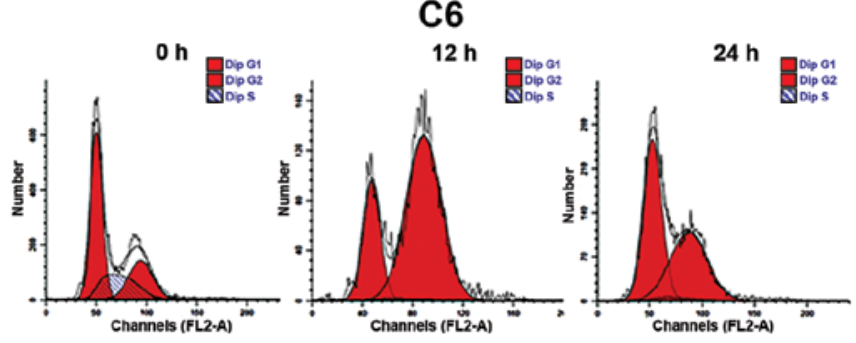

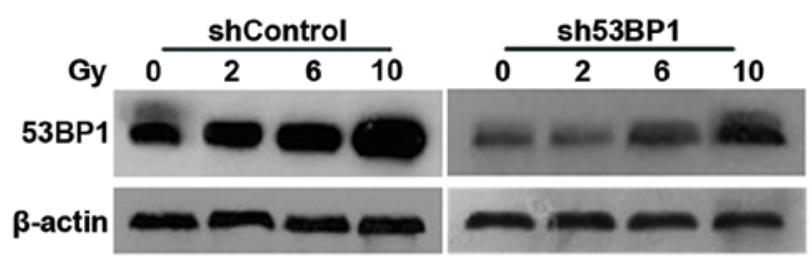

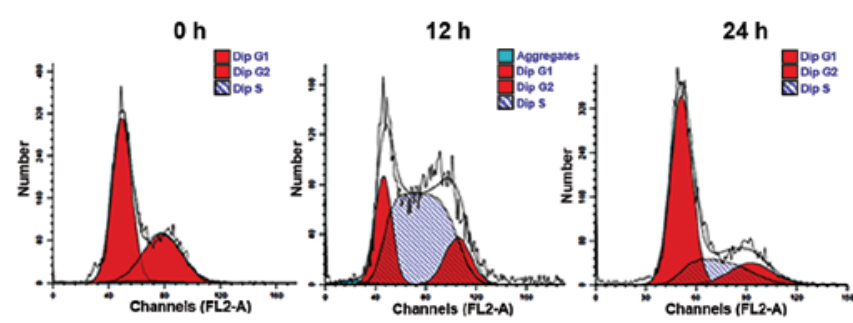

P6
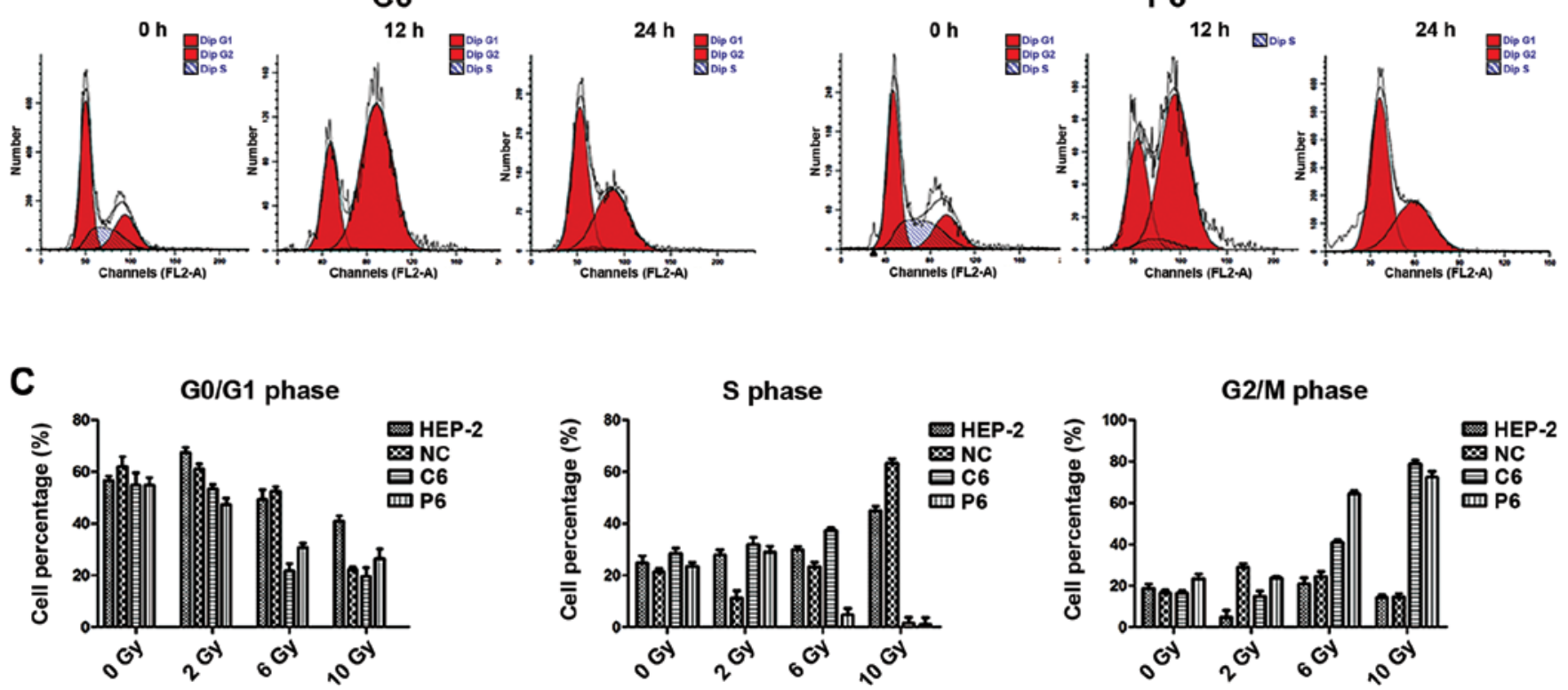

Figure 4. Cell cycle differences among cell lines were detected by western blotting and FCM. (A) The change of the protein level $12 \mathrm{~h}$ after different doses of IR exposure. (B) Cell cycle distribution in different cell lines under 10 Gy exposure after 0,12 , and 24 h. (C) Cell cycle distribution induced by unirradiated, 2,6 , and 10 Gy dose of IR after $12 \mathrm{~h} .{ }^{*} \mathrm{P}<0.05$. IR, ionizing radiation; FCM, flow cytometry.

The use of IR in radiotherapy is based on the fact that exposure to direct radiation may lead to significant levels of DNA damage, such as complex DSB, resulting in loss of clonogenic survival and tumor cell death. DDR may be a barrier of effective cell death for radiotherapy (26). As important proteins of DDR, their role in cell radioresistance was determined using the colony formation assay. A smaller population of C6 and P6 cells sustained an ability to form colony following treatment with IR compared with the parental and shNC-transfected cells at each X-ray dose. The results from the analysis of the radiation dose-related survival curves showed significant differences in the radiation indicators between the cells with stable MDC1- and 53BP1-inhibition and the parental cells. A distinct reduction in the D0 of C6 and P6 cells compared with the control group cells was found, suggesting that the downregulation of MDC1 and 53BP1 potentially enhancea radiosensitivity. Dq, an indicator exhibiting the cell ability to recover from radio-induced damage, was markedly decreased in the MDC1- and 53BP1-inhibited Hep-2 cells. SF2, another important indicator of radiotherapy, was found to decrease significantly in the MDC1- and 53BP1-inhibited cells, indicating that $\mathrm{MDC1}$ and 53BP1 inhibition may be appropriate for enhancing the effectiveness of fractionated radiotherapy (CFRT) and even hyper-fraction radiotherapy (HFRT) (27-29). The results therefore show that the low expression levels of MDC1 and 53BP1 promoted tumor cell sensitivity to radiotherapy. The finding that a combination of IR treatment with downregulation of MDC1 and 53BP1 expression to achieve a more effective radiotherapy is a novel strategy.

Cell cycle arrest causes delays in cell cycle progression and the co-ordination of DNA repair. In the present study, we detected a notable change in cell cycle distribution. A robust accumulation of $\mathrm{G} 2 / \mathrm{M}$ phase cells was observed in the MDC1and 53BP1-inhibited cells after $12 \mathrm{~h}$ of exposure to 6 and $10 \mathrm{~Gy}$ 
A
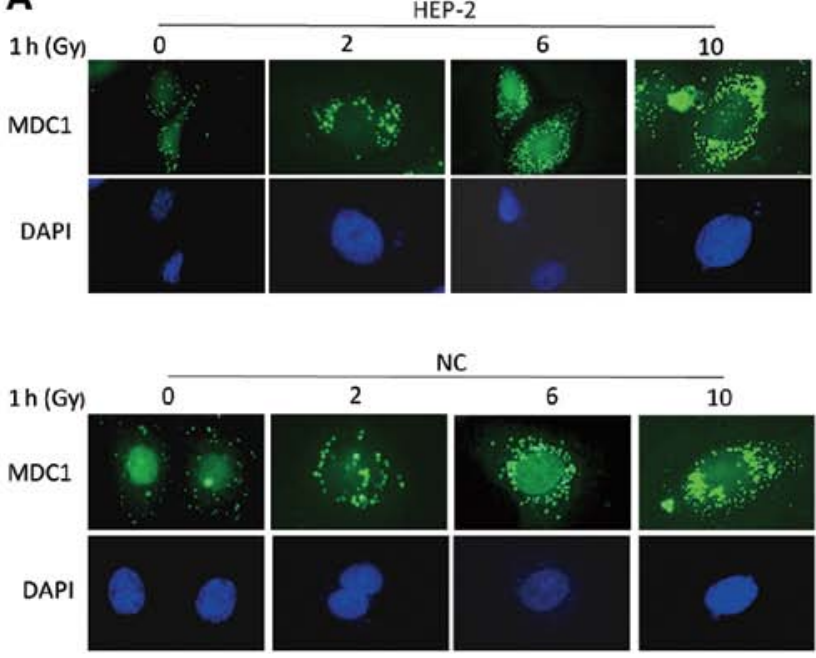

C6
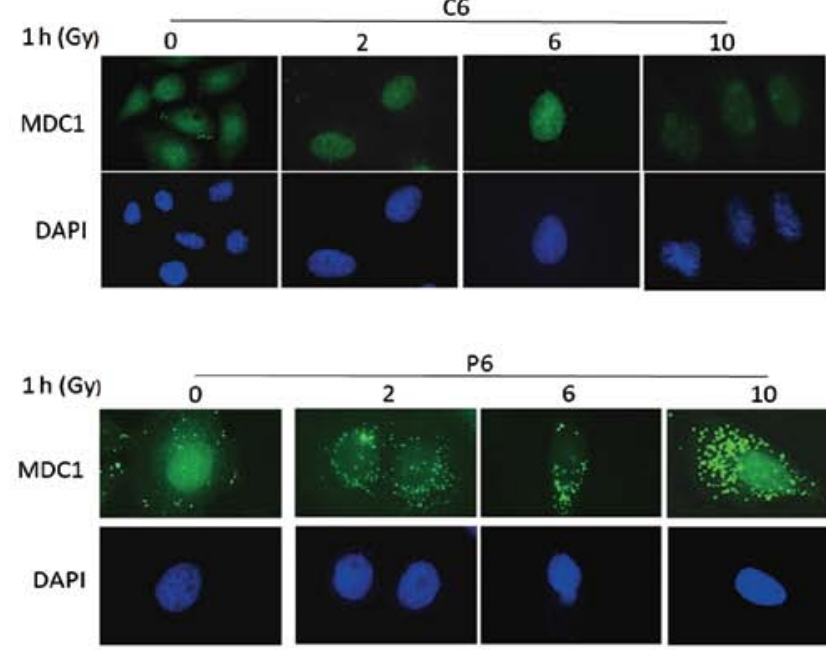
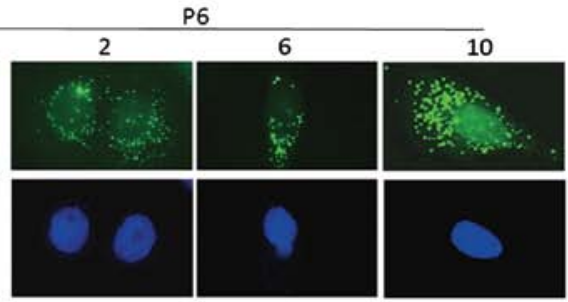

B
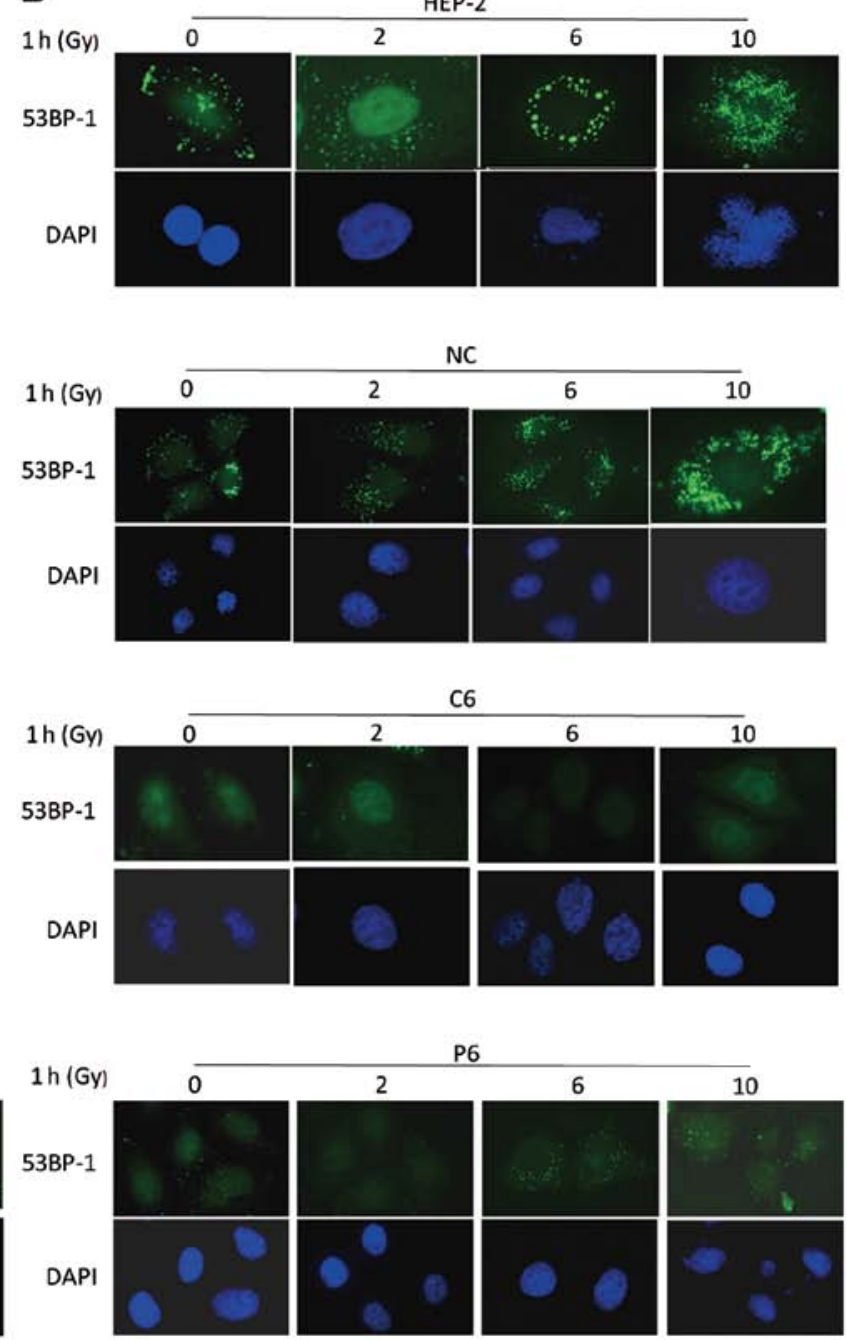

Figure 5. The foci of different cell lines measured using a laser confocal microscope at $1 \mathrm{~h}$ after IR. (A) Hep-2, NC, C6 and P6 cell lines were exposed to 0 , 2, 6 and $10 \mathrm{~Gy} \mathrm{IR}$ and incubated at $37^{\circ} \mathrm{C}$ for $1 \mathrm{~h}$ before staining for MDC1 (green). DNA was counterstained with DAPI (blue). (B) The same cell lines were exposed to $0,2,6$ and $10 \mathrm{~Gy}$ and incubated at $37^{\circ} \mathrm{C}$ for $1 \mathrm{~h}$ before staining for 53BP1 (green). DNA were counterstained with DAPI (blue). IR, ionizing radiation; MCD1, mediator of DNA damage checkpoint 1; NC, negative control.

IR. Twenty-four hours after IR treatment, the parental and shNC-transfected cells returned to the cell cycle distribution, which was identical to that of the corresponding non-IR-treated cells. However, no difference in the cell cycle distribution of C6 and P6 between the cell populations analyzed 12 or $24 \mathrm{~h}$ after being subjected to X-rays was detected. It indicated that $\mathrm{MDC1}$ and 53BP1 did not play a key role in delaying G2/M cell progression. Of note, a significant decrease of intra-S-phase cells was detected after 6 Gy treatment in P6 cells and the same reduction was evident in C6 cells after exposure to $10 \mathrm{~Gy}$ IR. Previous studies verified that cells lacking functional 53BP1 showed a deficiency in the intra-S-phase-checkpoint. However, our results show that MDC1 and 53BP1 are important in the intra-S-phase checkpoint activated by the DNA damage, notably induced by high-dose IR $(20,30,31)$.

The expression of MDC1 and 53BP1 in C6 and P6 cells was detected following IR treatment. Our results show that the two proteins increased in an IR dose-dependent manner, which coincided with the robust accumulation of G2/M phase cells via an IR dose-dependent increase. The G2/M checkpoint prevented the cells from initiating mitosis when undergoing
DNA damage during G2, S and G1 phase, and provided cells with additional time for the repair of persistent DNA lesions. The 53BP1 and MDC1 proteins were effectively downregulated, however, an efficient G1 and G2/M DNA damage checkpoint response occurred. This result indicated a rare correlation of $\mathrm{G} 1$ and $\mathrm{G} 2 / \mathrm{M}$ checkpoint activation, maintained with the expression of MDC1 and 53BP1 (31). By contrast, recovery of the checkpoint is dependent on DNA repair. Thus, extension of the checkpoint duration may be caused by the failure of DNA repair (9).

IR-induced foci formation is the result of the increased concentration of DDR proteins at the DNA damage site (13) and is a highly regulated process. We observed that the low accumulation of 53BP1 to the foci in the cells with MDC1 was downregulated. MDC1, as the master organizer of protein assembly at irradiation-induced foci (IRIF), is important for the recruitment of many downstream IRIF-associated factors, such as NBS1 and RNF8 $(4,9)$. Previous studies have reported that 53BP1 directly binds to phosphorylated $\mathrm{H} 2 \mathrm{~A}$. In the present study, the results show that 53BP1 was recruited to sites of DNA DSBs with an MDC1 dose-dependent manner (32). Of note, the 
formation of foci seems irrelevant with regard to $\mathrm{G} 1$ and G2/M checkpoint activation and maintenance because of fewer 53BP1 and MDC1 foci in the 53BP1- and MDC1-inhibited cells.

We conclude that $\mathrm{MDC} 1$ and 53BP1 are important in tumor cell resistance to radiotherapy and it is a potential strategy, which combines IR treatment through the downregulation of MDC1 and 53BP1 with special antibodies or shRNA, to obtain a more efficient radiotherapy.

\section{Acknowledgements}

This study was financially supported by Sichuan Support Project of Science and Technology (no. 2009SZ0160).

\section{References}

1. Giglia-Mari G, Zotter A and Vermeulen W: DNA damage response. Cold Spring Harb Perspect Biol 3: a000745, 2011.

2. Nakamura TM, Du LL, Redon C and Russell P: Histone H2A phosphorylation controls Crb2 recruitment at DNA breaks, maintains checkpoint arrest, and influences DNA repair in fission yeast. Mol Cell Biol 24: 6215-6230, 2004.

3. Ismail IH, Nyström S, Nygren J and Hammarsten O: Activation of ataxia telangiectasia mutated by DNA strand break-inducing agents correlates closely with the number of DNA double strand breaks. J Biol Chem 280: 4649-4655, 2005.

4. Bencokova Z, Kaufmann MR, Pires IM, Lecane PS, Giaccia AJ and Hammond EM: ATM activation and signaling under hypoxic conditions. Mol Cell Biol 29: 526-537, 2009.

5. Rube CE, Zhang S, Miebach N, Fricke A and Rube C: Protecting the heritable genome: DNA damage response mechanisms in spermatogonial stem cells. DNA Repair (Amst) 10: 159-168, 2011.

6. Dimitrova $\mathrm{N}$ and de Lange T: MDC1 accelerates nonhomologous end-joining of dysfunctional telomeres. Genes Dev 20 : 3238-3243, 2006.

7. Dimitrova N, Chen YC, Spector DL and de Lange T: 53BP1 promotes non-homologous end joining of telomeres by increasing chromatin mobility. Nature 456: 524-528, 2008.

8. Chitnis MM, Lodhia KA, Aleksic T, Gao S, Protheroe AS and Macaulay VM: IGF-1R inhibition enhances radiosensitivity and delays double-strand break repair by both non-homologous end-joining and homologous recombination. Oncogene, 5262-5273, 2014.

9. Huen MS, Grant R, Manke I, Minn K, Yu X, Yaffe MB and Chen J: RNF8 transduces the DNA-damage signal via histone ubiquitylation and checkpoint protein assembly. Cell 131: 901-914, 2007.

10. Moon SH, Nguyen TA, Darlington Y, Lu X and Donehower LA: Dephosphorylation of $\gamma$-H2AX by WIP1: an important homeostatic regulatory event in DNA repair and cell cycle control. Cell Cycle 9: 2092-2096, 2010.

11. Townsend PA, Cragg MS, Davidson SM, McCormick J, Barry S, Lawrence KM, Knight RA, Hubank M, Chen PL, Latchman DS et al: STAT-1 facilitates the ATM activated checkpoint pathway following DNA damage. J Cell Sci 118: 1629-1639, 2005.

12. Melander F, Bekker-Jensen S, Falck J, Bartek J, Mailand N and Lukas J: Phosphorylation of SDT repeats in the MDC1 N terminus triggers retention of NBS1 at the DNA damage-modified chromatin. J Cell Biol 181: 213-226, 2008.

13. Lin SY, Liang Y and Li K: Multiple roles of BRIT1/MCPH1 in DNA damage response, DNA repair, and cancer suppression. Yonsei Med J 51: 295-301, 2010.

14. Cho YW, Hong T, Hong S, Guo H, Yu H, Kim D, Guszczynski T, Dressler GR, Copeland TD, Kalkum M, et al: PTIP associates with MLL3- and MLL4-containing histone H3 lysine 4 methyltransferase complex. J Biol Chem 282: 20395-20406, 2007.
15. Kumagai A, Lee J, Yoo HY and Dunphy WG: TopBP1 activates the ATR-ATRIP complex. Cell 124: 943-955, 2006.

16. Tusher VG, Tibshirani R and Chu G: Significance analysis of microarrays applied to the ionizing radiation response. Proc Natl Acad Sci USA 98: 5116-5121, 2001.

17. Falck J, Petrini JH, Williams BR, Lukas J and Bartek J: The DNA damage-dependent intra-S phase checkpoint is regulated by parallel pathways. Nat Genet 30: 290-294, 2002.

18. Quanz M, Chassoux D, Berthault N, Agrario C, Sun JS and Dutreix M: Hyperactivation of DNA-PK by double-strand break mimicking molecules disorganizes DNA damage response. PLoS One 4: e6298, 2009.

19. Franken NA, Rodermond HM, Stap J, Haveman J and van Bree C: Clonogenic assay of cells in vitro. Nat Protoc 1: 2315-2319, 2006.

20. Bartkova J,HorejsíZ, Sehested M, Nesland JM, Rajpert-De Meyts E, Skakkebaek NE, Stucki M, Jackson S, Lukas J and Bartek J: DNA damage response mediators MDC1 and 53BP1: constitutive activation and aberrant loss in breast and lung cancer, but not in testicular germ cell tumours. Oncogene 26: 7414-7422, 2007.

21. Li X, Corsa CA, Pan PW, Wu L, Ferguson D, Yu X, Min J and Dou Y: MOF and H4 K16 acetylation play important roles in DNA damage repair by modulating recruitment of DNA damage repair protein Mdc1. Mol Cell Biol 30: 5335-5347, 2010.

22. Yamauchi M, Oka Y, Yamamoto M, Niimura K, Uchida M, Kodama S, Watanabe M, Sekine I, Yamashita S and Suzuki K: Growth of persistent foci of DNA damage checkpoint factors is essential for amplification of G1 checkpoint signaling. DNA Repair (Amst) 7: 405-417, 2008.

23. Park I and Avraham HK: Cell cycle-dependent DNA damage signaling induced by ICRF-193 involves ATM, ATR, CHK2, and BRCA1. Exp Cell Res 312: 1996-2008, 2006.

24. Ryu JS, Kang SJ and Koo HS: The 53BP1 homolog in C.elegans influences DNA repair and promotes apoptosis in response to ionizing radiation. PLoS One 8: e64028, 2013.

25. Lou Z, Minter-Dykhouse K, Wu X and Chen J: MDC1 is coupled to activated CHK2 in mammalian DNA damage response pathways. Nature 421: 957-961, 2003.

26. Rodier F, Muñoz DP, Teachenor R, Chu V, Le O, Bhaumik D, CoppéJP,Campeau E, Beauséjour CM,Kim SH, et al: DNA-SCARS: distinct nuclear structures that sustain damage-induced senescence growth arrest and inflammatory cytokine secretion. J Cell Sci 124: 68-81, 2011

27. Miyakawa A, Shibamoto Y, Otsuka S and Iwata H: Applicability of the linear-quadratic model to single and fractionated radiotherapy schedules: an experimental study. J Radiat Res 55: 451-454, 2013

28. Kajanti M, Blomqvist C, Lehtonen H, Kouri M, Wiklund T and Holsti LR: Biweekly dose escalation in curative accelerated hyperfractionation for advanced head and neck cancer: a feasibility study. Int J Radiat Oncol Biol Phys 39: 837-840, 1997.

29. Wang JH, Lu XJ, Zhou J and Wang F: A randomized controlled trial of conventional fraction and late course accelerated hyperfraction three-dimensional conformal radiotherapy for esophageal cancer. Cell Biochem Biophys 62: 107-112, 2012.

30. Eliezer Y, Argaman L, Rhie A, Doherty AJ and Goldberg M: The direct interaction between 53BP1 and MDC1 is required for the recruitment of 53BP1 to sites of damage. J Biol Chem 284: 426-435, 2009.

31. Shibata A, Barton O, Noon AT, Dahm K, Deckbar D, Goodarzi AA, Löbrich M and Jeggo PA: Role of ATM and the damage response mediator proteins 53BP1 and MDC1 in the maintenance of G(2)/M checkpoint arrest. Mol Cell Biol 30: 3371-3383, 2010.

32. Lee H, Kwak HJ, Cho IT, Park SH and Lee CH: S1219 residue of 53BP1 is phosphorylated by ATM kinase upon DNA damage and required for proper execution of DNA damage response. Biochem Biophys Res Commun 378: 32-36, 2009. 